Reprod. Nutr. Dévelop., 1980, 20 (3 A), 637-645.

\title{
Synthèse des triglycérides et catabolisme de l'acide oléique par les microsomes et les mitochondries hépatiques de rats traités par le clofibrate ou le procétofène
}

\author{
par Edith CLOUET *, R. PARIS * \\ avec la collaboration technique de Marguerite-Marie BOUTILLON * et de P. CLOUET * \\ * Laboratoire de Physiologie animale ef de la Nutrifion, UER Nutrition 21004 Dijon Cédex.
}

Summary. Triglyceride synthesis and oleic acid catabolism by hepatic microsomes and mitochondria in clofibrate or procetofen-treated rats.

Several studies have explained the influence of clofibrate on lipid metabolism, and the depressive effect of this drug on blood triglyceride and cholesterol levels is now well-known. Inhibition of triglyceride formation in the liver, and thus a reduced release of newly synthesized triglycerides coupled with increased fatty acid catabolism, might partially explain the lipid-lowering effect of clofibrate.

These in vitro studies were designed to determine the effects of a new normolipemic product, procetofen, on hepatic microsomal synthesis of triglycerides and mitochondrial catabolism of oleic acid. These subcellular particles were isolated from normal rats or those treated for 8 days with clofibrate $(250 \mathrm{mg} / \mathrm{kg} /$ day $)$ or procetofen $(100 \mathrm{mg} / \mathrm{kg} / \mathrm{day})$.

Microsomal triglyceride synthesis from ${ }^{3} \mathrm{H}$ oleate was not significantly changed in the clofibrate-treated group, while it decreased by 25 p. 100 in the procetofen-treated group.

In the mitochondrial system, ${ }^{14} \mathrm{CO}_{2}$ production from ${ }^{10-14} \mathrm{C}$ oleate was small and unaffected by either drug. Therefore, the radioactivity of acid-soluble products was enhanced by 40 or 130 p. 100 when mitochondria were isolated from the livers of rats treated with clofibrate or procetofen, respectively.

These data support the hypothesis that the plasma lipid-lowering effect of procetofen in vivo could be explained by increased hepatic fatty acid degradation which probably induced a reduction of triglyceride synthesis.

\section{Introduction.}

L'emploi du Clofibrate (chlorophénoxy-isobutyrate d'éthyle) ou du Procétofène (ester isopropylique de l'acide méthyl-2 [(chloro 4'-benzoyl)-4 phénoxy]-2 propionique) provoque un abaissement du taux de lipides sanguins chez l'Homme hyperlipémique (Thorp, 1966 ; Hess ef al., 1967 ; Cenedella, 1972 ; Gurrieri ef al., 1976 ; Rouffy et al., 1976 ; Wülfert ef al., 1976). Chez le rat normolipémique, ces produits entraînent une baisse des quantités de triglycérides sanguins nouvellement synthétisés par le foie à partir d'acide oléique et de glycérol marqués (Clouet et Paris, 1974, 1975). 
Cette diminution pourrait résulter de diverses modifications au niveau hépatique; ainsi une moindre synthèse lipidique par le réticulum endoplasmique et (ou) une oxydation accrue des acides gras sont deux phénomènes susceptibles de justifier l'effet hypotriglycéridémiant de ces produits.

Depuis 1962, de nombreuses études ont tenté d'expliquer l'action hypolipémiante du Clofibrate, et il est maintenant bien connu que cette drogue modifie l'activité de multiples systèmes enzymatiques intervenant dans le métabolisme des lipides. C'est ainsi qu'elle provoque, en particulier au niveau hépatique, une inhibition de la synthèse d'acides gras à partir de l'acétate (Maragoudakis, 1969 ; Maragoudakis ef Hankin, 1971 ; Havel et Kane, 1973 ; Homcy et Margolis, 1974 ; Landriscina ef al., 1975, 1977) mais aussi une accélération de leur catabolisme (Solberg ef al., 1972 ; Daae ef Aas, 1973 ; Moody and Reddy, 1974 ; Kahönen, 1976). Pour de nombreux auteurs, (Adams et al., 1971 ; Fallon et al., 1972 ; Brindley et Bowley, 1975 ; Clouet et Paris, 1974 ; Cenedella et Crouthamel, 1976) le Clofibrate entraînerait surtout une diminution de la synthèse hépatique des triglycérides.

Le Procétofène, produit normolipémiant commercialisé depuis 1975, a par contre fait l'objet de très peu d'études concernant le métabolisme des triglycérides. Celles-ci font état de ses effets sur les paramètres lipidiques chez le rat (Gurrieri et al., 1976) et chez l'Homme (Fromantin et al., 1977). A l'heure actuelle, le mode d'action du Procétofène sur le métabolisme des triglycérides n'est pas encore complètement élucidé.

II nous a donc paru intéressant de comparer in vitro, les effets de l'administration chronique du Procétofène à ceux du Clofibrate, d'une part sur la synthèse des triglycérides et, d'autre part sur le catabolisme de l'acide oléique dans le foie. Nous avons choisi cet acide gras, car bien représenté parmi les acides gras libres plasmatiques (Dittmer ef Hanahan, 1959 ; Clovet ef Paris, 1974, 1975), il constitue aussi 30 p. 100 des acides gras des triglycérides hépatiques; il est incorporé surtout dans les triglycérides hépatiques par rapport aux phospholipides.

\section{Matériel et méthodes.}

A. - Produits utilisés. - L'acide oléique 9-10 ${ }^{3} \mathrm{H}_{2}$ ou $10{ }^{14} \mathrm{C}$ (CEA Saclay, France) radiochimiquement pur à 99 p. 100 est testé puis purifié avant utilisation. Les autres composés chimiques et les cofacteurs d'incubation sont des produits purs (Sigma, Merck ou Prolabo).

B. - Animaux. - Nous avons utilisé des rats mâles adultes, de Souche Wistar (Elevage de Lessieux, 95 Brayleu).

Les animaux, pesant $210 \pm 20 \mathrm{~g}$ sont séparés en quatre lots $(H, C, E, P)$ et sont nourris ad libifum avec un régime équilibré (référence $A . O_{4}$ ) fourni par UAR (Villemoisson/Orge). Pendant les huit jours précédant l'expérience, les rats reçoivent quotidiennement par intubation gastrique et pour $100 \mathrm{~g}$ de poids corporel, $0,5 \mathrm{ml}$ des solutions suivantes :

lot $\mathrm{H}$ : huile de tournesol ;

lot $C$ : Clofibrate à 5 p. 100 dans l'huile de tournesol, de telle sorte que chaque animal reçoive $250 \mathrm{mg} / \mathrm{kg} / \mathrm{jour}$ de produit ; 
lot $E$ : eau gommeuse (gomme arabique Prolabo) à 3 p. 100 dans l'eau distillée ; lot $P$ : Procétofène à 2 p. 100 en suspension dans l'eau gommeuse de telle sorte que chaque animal reçoive $100 \mathrm{mg} / \mathrm{kg} /$ jour de produit.

\section{C. - Techniques.}

a) Obiention des fractions subcellulaires. - Après un jeûne hydrique de $18 \mathrm{~h}$, les animaux sont sacrifiés par décapitation ; le foie est prélevé puis abondamment perfusé ef rincé à l'aide de sérum physiologique glacé ; il est ensuite séché et pesé. On le plonge alors dans une solution de saccharose $0,3 \mathrm{M}$. Après 4 min d'homogénéisation, à 2500 tours/min dans un cylindre de Potter, la suspension de foie est diluée par du saccharose $0,3 \mathrm{M}$ glacé, de telle sorte que $9 \mathrm{ml}$ de cette suspension contiennent $1 \mathrm{~g}$ de foie.

La séparation des fractions subcellulaires est réalisée selon une méthode inspirée de Sedgwick et Hübscher (1965). Les mitochondries sont obtenues par centrifugation à $4300 \mathrm{~g}$ pendant $10 \mathrm{~min}$; les microsomes sont isolés par centrifugation du surnageant à $104000 \mathrm{~g}$ pendant $1 \mathrm{~h}$ (centrifugeuse Spinco L2 65B). Dans chaque cas, les culots «mitochondries » et « microsomes» sonł à nouveau homogénéisés dans du saccharose $0,3 \mathrm{M}$ ef soumis à une nouvelle centrifugation identique, ces opérations étant effectuées à une température comprise entre 1 et $4^{\circ} \mathrm{C}$. Les culołs ainsi obtenus sont remis en suspension dans un mélange tampon phosphate de $\mathrm{pH} 7,4(11 \mathrm{mM}$ ) (à raison de 2 vol. de solution tampon pour 1 vol. de fractions subcellulaires). La teneur protéique des préparations est déterminée par dosage spectrophotométrique selon la méthode de Layne (1957) ; connaissant la concentration, on détermine le volume de la partie aliquote nécessaire pour obtenir la quantité de protéines choisie pour l'incubation.

b) Conditions d'incubation.

- Incubations microsomales. - La composition du milieu d'incubation microsomale, directement inspirée de Smith ef Hübscher (1966) est choisie de façon à obtenir l'incorporation maximale de l'acide oléique dans les triglycérides (voir fig. 1). Les incubations, réalisées à $37^{\circ} \mathrm{C}$ sous agitation permanente sont arrêtées à des temps variables par addition de $0,5 \mathrm{ml}$ de $\mathrm{HCl} 6 \mathrm{~N}$ et de méthanol. Après extraction des lipides totaux, selon la méthode de Delsal (1944), les triglycérides sont séparés par chromatographie sur plaques de verre, recouvertes d'une couche de $0,5 \mathrm{~mm}$ d'épaisseur de gel de silice $G$ Merck. L'élution est réalisée à l'aide d'un mélange hexane, éther sulfurique, acide acétique $(90 / 30 / 1 ; V / V / V)$. Les constituants lipidiques sont révélés par des vapeurs d'iode ; après disparition de la coloration, le gel de silice supportant les triglycérides est transféré dans un pot à scintillation, l'élution des triglycérides étant assurée par $1 \mathrm{ml}$ de méthanol RP.

- Incubations mitochondriales. - La composition du milieu d'incubation est directement inspirée de la technique mise au point par Blond ef al. (1975) (fig. 2). L'incubation est réalisée sous agitation permanente à $37^{\circ} \mathrm{C}$ dans un récipient fermé comportant 2 compartiments; le gaz carbonique libéré est capté par $0,3 \mathrm{ml}$ d'hydroxyde de hyamine (Packard, France) placé dans le compartiment central. Après $30 \mathrm{~min}$, les réactions sont arrêtées par l'addition de $0,1 \mathrm{ml}$ de $\mathrm{HCl} 10 \mathrm{~N}$; l'incubation est ensuite prolongée pendant 30 min afin que la totalité du $\mathrm{CO}_{2}$ soit dégagée et captée. Après ce 
temps, le milieu d'incubation est acidifié par de l'acide perchlorique à 10 p. 100 , puis filtré sur filtre «Millipore » (pores de $0,45 \mu$ de diamètre). Les produits résultant de l'oxydation de l'acide oléique, solubles en milieu acide (molécules courtes issues de la $\beta$-oxydation, différents acides du cycle de Krebs, produits intermédiaires ef finaux de la cétogenèse) sont contenus dans le filtrat que nous appelerons « phase acido-soluble $)$.

c) Mesure des radioactivités. - La radioactivité des triglycérides synthétisés ef du gaz carbonique dégagé est mesurée, après adjonction de Permafluor III (Packard), à l'aide d'un spectromètre à scintillation liquide Packard 3380. La radioactivité des produits acido-solubles est déterminée en ajoutant $10 \mathrm{ml}$ d'lnstagel (Packard) à $1 \mathrm{ml}$ de solution aqueuse.

d) Analyse statistique. - Pour chaque temps et chaque lot les incubations microsomales et mitochondriales ont été réalisées respectivement en 4 et 3 exemplaires; les microsomes et les mitochondries utilisés au cours de chaque essai provenant du foie d'animaux distincts.

Nos résultats sont exprimés par la moyenne des valeurs obtenues à chaque essai. Dans le cas de comparaison de moyennes, la valeur de la probabilité p a été calculée à partir du test de Student.

\section{Résultats.}

Quel que soit le lot considéré, la synthèse des triglycérides est rapide mais faible ; les proportions d'acide oléique marqué incorporé dans cette fraction représentent 1 à 1,5 p. 100 de la radioactivité initiale (fig. $1 \mathrm{~A}$ et $\mathrm{B}$ ).
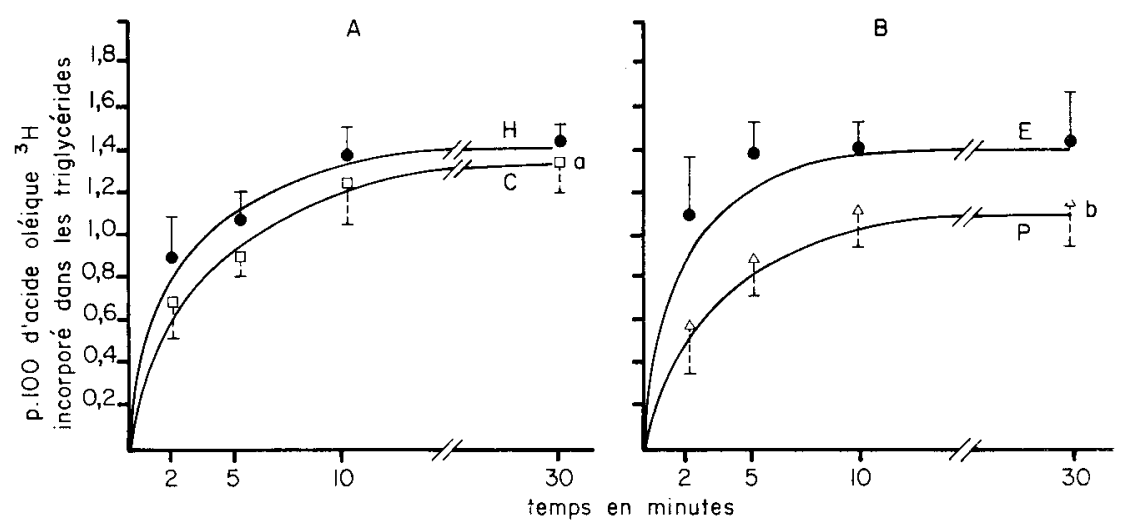

FIG. 1. - Evolution dans le temps des quantités d'acide oléique ${ }^{3} \mathrm{H}$ incorporées dans les triglycérides (en p. 100 de la radioactivité initiale présente dans l'incubat). • : microsomes hépatiques d'animaux des lots témoins (H-E) ; $\square$ : microsomes hépatiques d'animaux du lot Clofibrate (C); $\triangle$ : microsomes hépatiques d'animaux du lot Procétofène $(\mathrm{P})$. Les valeurs représentées correspondent à la moyenne arithmétique des résultats de quatre incubations, effectuées à partir de foie d'animaux distincts. ( $a: N S ; b: p<0,05$ ). Milieu d'incubation : 6,6 mM d'ATP; $0,033 \mathrm{mM}$ de CoA ; $20 \mathrm{mM}$ de glycérol ; 6,6 mM de KF ; $6,6 \mathrm{mM}$ de $\mathrm{MgCl}_{2} ; 0,8 \mathrm{mM}$ d'oléate de potassium $9-10{ }^{3} \mathrm{H}_{2}(157$ $\mu \mathrm{Ci} / \mathrm{mM}) ; 2 \mathrm{mg}$ de protéines microsomales, tampon phosphate $\mathrm{pH} 7,4$ Qsp $3 \mathrm{ml}$. 
Le traitement par le Clofibrate ne modifie pas significativement les taux d'incorporation de l'acide oléique dans les triglycérides (fig. 1A) ; par contre, l'administration de Procétofène entraîne une diminution de 25 p. 100 en moyenne des proportions observées chez les témoins $(\mathrm{P}<0,05)$ (fig. 1B).

L'étude du catabolisme de l'acide oléique $10{ }^{14} \mathrm{C}$, par les mitochondries de foie, révèle une production de ${ }^{14} \mathrm{CO}_{2}$ très faible (en moyenne $0,1 \mathrm{p} .100$ de la radioactivité initiale), quel que soit le lot dont sont issues les mitochondries (fig. 2).

Lorsque les mitochondries sont isolées du foie d'animaux traités par le Procétofène, la production du ${ }^{14} \mathrm{CO}_{2}$ augmente de 32 p. 100 par rapport à celle observée chez les témoins, aucune modification significative n'étant constatée à partir des mitochondries hépatiques de rats traités par le Clofibrate.

Par contre, la radioactivité des produits acido-solubles du catabolisme de l'acide oléique (voir fig. 3) est nettement plus élevée que celle du gaz carbonique dégagé ; elle atteint en effet environ 9,6 p. 100 de la radioactivité initiale de l'acide oléique lorsque les mitochondries sont isolées du foie d'animaux témoins. Après traitement par le Clofibrate ou le Procétofène, le marquage des produits acido-solubles représente respectivement 13,6 et 21,5 p. 100 de la radioactivité initiale.

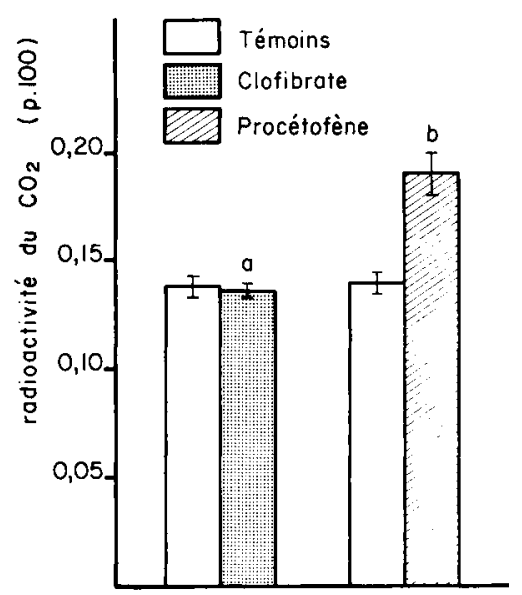

FIG. 2 .

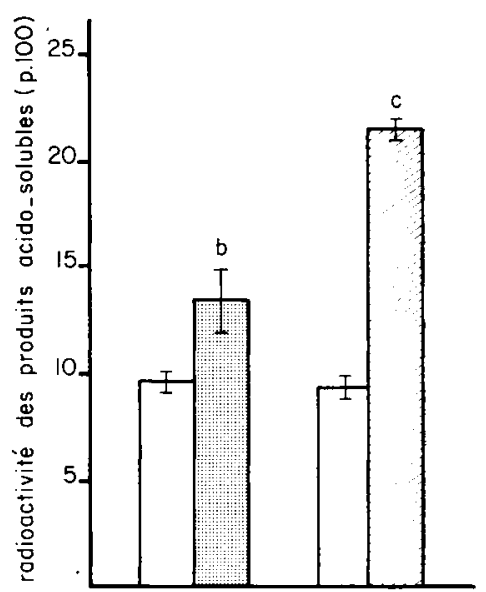

FIG. 3.

FIG. 2. - Radioactivité du $\mathrm{CO}_{2}$ produit pendant $30 \mathrm{~min}$ lors du catabolisme de l'acide oléique $10^{14} \mathrm{C}$ par $1 \mathrm{mg}$ de protéines mitochondriales de foie d'animaux témoins ou traités par le Clofibrate ou le Procétofène (en p. 100 de la radioactivité de l'acide oléique $10^{14} \mathrm{C}$ initialement présent dans l'incubation). Ces valeurs correspondent à la moyenne arithmétique des résultats obtenus au cours de trois. incubations ( $a, b$ : voir fig. 1). Milieu d'incubation : $50 \mathrm{mM} \mathrm{de} \mathrm{KCl} ; 4 \mathrm{mM} \mathrm{de} \mathrm{MgCl}$, ; $1 \mathrm{mM} \mathrm{d}$ 'ATP ; $1 \mathrm{mM}$ de DL-carnitine ; $20 \mu \mathrm{M}$ de CoA ; $50 \mu \mathrm{M}$ d'oléate de potassium $10{ }^{14} \mathrm{C}(1,35 \mu \mathrm{Ci} / \mathrm{mM})$; un volume de suspension mitochondriale représentant $1 \mathrm{mg}$ de protéines; tampon phosphate $\mathrm{pH} 7,4$ QSP $2 \mathrm{ml}$; température $37^{\circ} \mathrm{C}$.

FIG. 3. - Radioactivité des produits acidosolubles issus du catabolisme de l'acide oléique $10{ }^{14} \mathrm{C}$ par $1 \mathrm{mg}$ de protéines mitochondriales de foie d'animaux témoins ou traités par le Clofibrate ou le Procétofène (conditions d'incubations indiquées sous la fig. 2). (b : $p<0,05 ; c: p<0,01$ ). 


\section{Discussion.}

Pour des quantités de protéines identiques, les microsomes hépatiques de rats normolipémiques et traités pendant 8 jours par le Clofibrate $(250 \mathrm{mg} / \mathrm{kg} / \mathrm{j})$ conservent par rapport à leurs témoins, la même aptitude à synthétiser les triglycérides à partir de l'acide oléique.

Cette constatation est en désaccord avec les résultats de Lamb et Fallon (1972), Adams ef al. (1971), qui indiquent une diminution de la synthèse in vitro des triglycérides hépatiques. Toutefois, leurs conditions expérimentales, très différentes des nôtres, peuvent expliquer cette divergence ; en effet, ces auteurs ajoutent directement le Clofibrate en quantités variables, dans le milieu d'incubation contenant un homogénat de foie et non des microsomes.

Le Procétofène administré pendant 8 jours à raison de $100 \mathrm{mg} / \mathrm{kg} / \mathrm{j}$ induit par contre une réduction de la synthèse des triglycérides par les microsomes hépatiques, ainsi que le suggèrent les premiers résultats de travaux réalisés in vivo (Clouet et Paris, 1975).

Après administration de l'une ou l'autre drogue, les mitochondries hépatiques provoquent, pour des quantités de protéines mitochondriales identiques, un catabolisme de l'acide oléique plus important que celui constaté à partir des mitochondries d'animaux témoins.

La radioactivité du $\mathrm{CO}_{2}$ dégagé augmente certes de 32 p. 100 après traitement par le Procétofène, mais le $\mathrm{CO}_{2}$ libéré par l'oxydation de l'acide oléique $10{ }^{14} \mathrm{C}$ représente seulement environ 0,15 p. 100 de la radioactivité initiale de cet acide gras ; de plus, le marquage de l'oléate étant situé en milieu de chaîne et le fonctionnement du cycle de Krebs étant limité dans l'hépatocyte (Bremer et Wojtczak, 1972), il nous semble justifié de ne pas prendre en considération l'augmentation du dégagement de $\mathrm{CO}_{2}$ après ce traitement. En conséquence, seule une augmentation de la cétogénèse, certes favorisée par l'état de jeûne préalable des animaux, seraił le principal facteur de l'élévation du catabolisme de l'acide oléique après administration de Clofibrate ou de Procétofène.

Cependant d'autres auteurs rapportent après traitement par le Clofibrate des variations significalives de la production hépatique de $\mathrm{CO}_{2}$ à partir de divers acides gras ; ainsi Cederbaum ef al. (1976), après traitement de 14 jours chez le rat, indiquent une diminution de la production du ${ }^{14} \mathrm{CO}_{2}$ provenant de l'oxydation du palmitate ou de l'octanoate. Par contre, lors d'incubations d'hépatocytes isolés du foie d'animaux traités 8 jours par le Clofibrate, Christiansen (1978) constatent une oxydation du palmitate doublée par rapport aux témoins.

Dans nos expériences, la radioactivité des produits acido-solubles résultant du catabolisme de l'acide oléique par les miłochondries hépatiques d'animaux traités par le Clofibrate augmente de 40 p. 100 par rapport à celle constatée chez les témoins. Ces résultats sont à rapprocher de ceux observés chez l'Homme (Wolfe ef al., 1973) et chez le rat (Burch et Curran, 1969 ; Avoy et al., 1965 et Christiansen, 1978), ces auteurs notant une élévation de la cétogenèse après administration quotidienne de cette drogue.

Après traitement par le Procétofène, les mitochondries ont très nettement accru 
leur capacité d'oxydation vis-à-vis de l'acide oléique ; en effet, la radioactivité des produits de la phase acido-soluble présente une augmentation de 130 p. 100 par rapport à celle mesurée chez les témoins. Il semble donc que le Clofibrate et le Procétofène ne présentent pas des modes d'action qualitativement différents en ce qui concerne le catabolisme de l'acide oléique. Cependant, pour des quantités de protéines microsomales et mitochondriales identiques à celles utilisées dans les incubations témoins, le Procétofène s'avère une drogue plus active que le Clofibrate : en effet, malgré une administration pondéralement inférieure, le Procétofène provoque au niveau hépatique une nette augmentation du catabolisme de l'acide oléique et une diminution de la synthèse des triglycérides, à partir de cet acide gras.

En conclusion, considérant les diverses modifications obtenues dans nos expériences in vitro, il esł possible d'admettre que ces variations soient en partie responsables de la moindre synthèse hépatique de triglycérides constatée in vivo (Clovet et Paris, 1974, 1975) et par voie de conséquence, de l'effet hypotriglycéridémiant des deux produits testés. On peut en effet penser que la synthèse hépatique soit diminuée, non seulement par réduction de l'activité du réticulum endoplasmique, mais surtout par une moindre disponibilité de l'acide oléique, celui-ci étant préférentiellement dirigé vers les voies de dégradation.

De plus, si l'on considère l'importante hypertrophie du foie constatée chez le rat après traitement de 8 jours par l'une ou l'autre drogue (Hess ef al., 1967 ; Adams et al., 1971 ; Solberg ef al., 1972 ; Daae et Aas, 1973 ; Clovet et Paris, 1974, 1975 ; Cenedella et Crouthamel, 1976) ainsi que la prolifération mitochondriale du foie (Ramakrishna Kurup ef al., 1970 ; Krishnakantha ef Ramakrishna Kurup, 1972), il est vraisemblable que les constatations que nous rapportons in vitro soient très amplifiées dans les conditions in vivo.

A l'accroissement de l'activité mitochondriale, il faut aussi ajouter l'éventualité d'une oxydation peroxisomale importante, ainsi que le suggèrent Christiansen, 1978 ; Sroboda et Azarnoff, 1971.

Reçu en juin 1979.

Accepté en octobre 1979.

Remerciements. - Le Clofibrate et le Procétofène ont été respectivement offerts par les Laboratoires Avlon (95 Enghien-les-Bains) et Fournier (21 Dijon) que nous remercions.

\section{Références}

ADAMS L. L., WEBB W. W., FALLON H. J., 1971. Inhibition of hepatic triglyceride formation by clofibrate. J. clin. Invest., 50, 11, 2339-2346.

AVOY D. R., SWYRYD A., GOULD R. G., 1965. Effects of $\alpha$-p-chlorophenoxyisobutyryl-ethyl ester (CPIB) with and without androsterone on cholesterol biosynthesis in rat liver. J. Lipid Res., 6, 369-376.

BLOND J. P., CLOUET P., LEMARCHAL P., 1975. Oxydation de l'acide érucique ef de l'érucyl-CoA par les mitochondries isolées de cœur de rat. Comparaison à l'acide oléique. Biochimie, 57, $361-367$.

BREMER J., WOJTCZAK A. B., 1972. Factors controlling the rate of fatty acid $\beta$-oxidation in rat liver mitochondria. Biochim. biophys. Acta, 280, 515-530.

BRINDLEY D. N., BOWLEY M., 1975. Drugs affecting the synthesis of glycerides and phospholipids in rat liver : the effects of Clofibrate, Halofenate, Fenfluramine, Amphetamine, Cincho- 
caïne, Chlorpromazine, Dimethylimipramine, Mepyramine and some of their derivatives. Biochem. J., 148, 461-469.

BURCH R. E., CURRAN G. L., 1969. Hepatic acetoacetyl-CoA deacylase activity in rats fed ethyl chlorophenoxyisobutyrate (CPIB). J. Lipid Res., 10, 668-673.

CEDERBAUM A. I., MADHAVAN T. V., RUBIN E., 1976. Effect of chronic clofibrafe administration on mitochondrial fatty acid oxidation. Biochem. Pharm., 25, 1285-1292.

DELSAL J., 1944. Nouveau procédé d'extraction des lipides du sérum par le méthylal. Applications aux microdosages du cholestérol total, des phosphoaminolipides et des protéides. Bull. Soc. Chim. biol., 26, 99-105.

CENEDELLA R. J., 1972. Clofibrate and Nafenopin : Effects on plasma clearance and tissue distribution of chylomicron triglyceride in the dog. Lipids, 7, 644-652.

CENEDELLA R. J., CROUTHAMEL W., 1976. Halofenate and Clofibrate : mechanism of triglyceridemic action in the rat. J. Lipid Res., 17, 156-166.

CHRISTIANSEN R. J., 1978. The effect of Clofibrate feeding on hepatic fatty acid metabolism. Biochim. biophys. Acto, 530, 314-324.

CLOUET E., PARIS R., 1974. Influence du clofibrate sur l'incorporation du glycérol et de l'acide oléique dans les lipides corporels du rat. J. Physiol., 69, 2, $240 \mathrm{~A}$.

CLOUET E., PARIS R., 1975. Action du LF 178 (Lipantyl) sur le métabolisme lipidique du rat. J. Physiol., 71, 2, 272 A.

DAAE L. N. W., AAS W., 1973. Fatty acid activation and acyltransfer in rat liver during clofibrate feeding. Atherosclerosis, 17, 389-400.

DITTMER J. C., HANAHAN D. J., 1959. Biochemistry of long chain fatty acids, I general observations. J. biol. Chem., 234, 1976-1982.

FALLON H. J., ADAMS L. L., LAMB R. G., 1972. A review of studies on the mode of action of Clofibrate and Betabenzalbutyrate. Lipids, 7, 106-109.

FROMANTIN M., GAUTIER D., BON R., 1977. Traitement des hyperlipidémies essentielles par le procétofène (à propos de cent observations), Sem. Hop. Ther., 53, no 5-6, 311-316.

GURRIERI J., LE LOUS M., RENSON F. J., TOURNE C., VOEGELIN H., MAJOIE B., WULFERT E., 1976. Antilipidemic drugs ; Part 2 : experimental study of a new potent hypolipidemic drug, isopropyl-[4'-(p-chlorobenzoyl)-2-phenoxy-2-methyl]-propionate : LF 178. Arzneimittelforsch. (Drug Res.), 26, 889-894.

HAVEL R. J., KANE J. P., 1973. Drugs and lipid metabolism. Pharmacol. Rev., 6567, 287-308.

HESS R., RIESS W., STÄUBLI W., 1967. Hepatic action of hypolipidaemic drugs : effect of ethylchlorophenoxyisobutyrate. Progr. Biochem. Pharmacal., 2, 325-336.

HOMCY C. J., MARGOLIS S., 1974. Comparison of the effects of clofibrate and halofenate (MK185 ) in isolated rat hepatocytes. Atherosclerosis, 19, 381-391.

KAHÖNEN M. T., 1976. Effect of clofibrate treatment on carnitine acyltransferases in different subcellular fractions of rat liver. Biochim. biophys. Acta, 428, 690-701.

KRISHNAKANTHA T. P., RAMAKRISHNA KURUP C. K., 1972. Increase in hepatic catalase and glycerophosphate dehydrogenase activities on administration of Clofibrate and Clofenapate to the rat. Biochem. J., 130, 167-175.

LAMB R. G., FALLON H. J., 1972. Inhibition of monoacylglycerophosphate formation by chlorophenoxyisobutyrate and $\beta$-benzalbutyrate. J. biol. Chem., 247, 1281-1287.

LANDRISCINA C., GNONI G. V., QUAGLIARIELLO E., 1975. On the mode of action of clofibrate on lipid metabolism. Inhibition of rat liver microsomal fatty acid synthesis. Biochem. Med., 12, 356-366.

LANDRISCINA C., RUGGIERO F., GNONI G. V., and QUAGLIARIELLO E., 1977. Reduced activity of hepatic microsomal fatty acid chain elongation synthesis in clofibrate fed rats. Bioch. Pharm., 26, 1401-1404.

LAYNE E., 1957. Spectrophotometric and turbidimetric method for measuring proteins, 447-454. In COLOWICK S. P., KAPLAN N. O., Methods in enzymology, Acad. Press, New York.

MARAGOUDAKIS M. E., 1969. Inhibition of hepatic acetyl coenzyme A carboxylase by hypolipidemic agents. J. biol. Chem., 244, 5005-5013.

MARAGOUDAKIS M. E., HANKIN H., 1971. On the mode of action of lipid lowering agents. Part 5 (Kinetics of the inhibition in vitro of rat acetyl coenzyme A carboxylase). J. biol. Chem., 246, 348-357. 
MOODY D. E., REDDY J. K., 1974. Increase in hepatic carnitine acyltransferase activity associated with peroxysomial proliferation induced by clofibrate. Res. Comm. Chem. Pathol. Pharmacol., 9, 501-510.

RAMAKRISHNA KURUP C. K., AITHAL H. N., RAMASARMA T., 1970. Increase in hepatic mitochondria on administration of ethyl $\alpha$ - $\beta$-chlorophenoxyisobutyrate to the rat. Biochem. $J$., 116, 773-779.

ROUFFY J., DREUX C., GOUSSAULT Y., DAKKAR R., RENSON F. J., 1976. Antilipidemic drugs Part 5 : Evaluation of the hypolipidemic effect of LF 178 in 191 patients affected by the atherogenic form of endogenous hyperlipoproteinemia (types Ila, llb and IV). Arzneimittelforsch. (Drug Res.), 26, 901-906.

SEDGWICK B., HÜBSCHER G., 1965. Metabolism of phospholipids ; IX : phosphatidate phosphohydrolase in rat liver. Biochim. biophys. Acta, 106, 63-77.

SMITH M. E., HÜBSCHER G., 1966. The biosynthesis of glycerides by mitochondria from rat liver. Biochem. I., 101, 308-316.

SOLBERG H. E., AAS M., DAAE L. N. W., 1972. The activity on the different carnitine acyltransferases in the liver of Clofibrate-fed rats. Biochim. biophys. Acto, 280, 434-439.

SVOBODA D. J., AZARNOFF D. L., 1971. Effects of selected hypolipidemic drugs on cell ultrastructure. Fed. Proc., 30, 3, 841-847.

THORP J. M., 1966. Modes et niveaux d'action du clofibrate. Symp. sur le clofibrate (chlorophénoxyisobutyrate d'éthyle). Société d'Athérosclérose : (séance du 15 mai 1965). Rev. Athérosclérose, suppl. 1, 2-11.

WOLFE B. M., KANE J. P., HAVEL R. J., BREWSTER H. P., 1973. Mechanism of the hypolipemic effect of clofibrate in post absorptive man. J. clin. Invest., 52, 2146-2159.

WÜLFERT E., MAJOIE B., DE CEAURRIZ A., 1976. Antilipidemic drugs ; Part 6 : LF 178 in man : A preliminary note on a multicenter investigation bearing on 393 subjects with pure or mixed forms of hyperlipidemia. Arzneimittelforsch. (Drug Res.), 26, 906-909. 